\title{
Long-term adenoma recurrence following wide-field endoscopic mucosal resection (WF-EMR) for advanced colonic mucosal neoplasia is infrequent: results and risk factors in 1000 cases from the Australian Colonic EMR (ACE) study
}

\author{
Alan Moss, ${ }^{1,2}$ Stephen J Williams, ${ }^{1}$ Luke F Hourigan, ${ }^{4}$ Gregor Brown, ${ }^{3,5}$ \\ William Tam, ${ }^{6}$ Rajvinder Singh, ${ }^{6}$ Simon Zanati, ${ }^{2,5}$ Nicholas G Burgess, ${ }^{1,7}$ \\ Rebecca Sonson, ${ }^{1}$ Karen Byth, ${ }^{8}$ Michael J Bourke ${ }^{1,7}$
}

For numbered affiliations see end of article.

\section{Correspondence to} Professor Michael J Bourke, Director of Gastrointestinal Endoscopy, Department of Gastroenterology and Hepatology, Westmead Hospital, c/- Suite 106a, 151-155 Hawkesbury Road, Westmead, Sydney, New South Wales 2143, Australia; michael@citywestgastro.com.au

Meeting Presentation: Oral presentations at: Digestive Disease Week, San Diego 2012, Australian

Gastroenterology Week, Adelaide 2012.

Received 24 June 2013 Revised 27 May 2014 Accepted 5 June 2014 Published Online First 1 July 2014

CrossMark

To cite: Moss A,

Williams $\mathrm{SJ}$, Hourigan $\mathrm{LF}$,

et al. Gut 2015;64:57-65.

\section{ABSTRACT}

Objective Wide-field endoscopic mucosal resection (WF-EMR) is an alternative to surgery for treatment of advanced colonic mucosal neoplasia up to $120 \mathrm{~mm}$ in size, but has been criticised for its potentially high recurrence rates. We aimed to quantify recurrence at 4 months (early) and 16 months (late) following successful WF-EMR and identify its risk factors and clinical significance.

Design Ongoing multicentre, prospective, intention-totreat analysis of sessile or laterally spreading colonic lesions $\geq 20 \mathrm{~mm}$ in size referred for WF-EMR to seven academic endoscopy units. Surveillance colonoscopy (SC) was performed 4 months (SC1) and 16 months (SC2) after WF-EMR, with photographic documentation and biopsy of the scar.

Results 1134 consecutive patients were enrolled when 1000 successful EMRs were achieved, of whom 799 have undergone SC1. 670 were normal. Early recurrent/ residual adenoma was present in $128(16.0 \%, 95 \% \mathrm{Cl}$ $13.6 \%$ to $18.7 \%)$. One case was unknown. The recurrent/residual adenoma was diminutive in $71.7 \%$ of cases. On multivariable analysis, risk factors were lesion size $>40 \mathrm{~mm}$, use of argon plasma coagulation and intraprocedural bleeding. Of 670 with normal SC1, 426 have undergone $\mathrm{SC2}$, with late recurrence present in 17 cases $(4.0 \%, 95 \% \mathrm{Cl} 2.4 \%$ to $6.2 \%)$. Overall, recurrent/ residual adenoma was successfully treated endoscopically in 135 of 145 cases $(93.1 \%, 95 \% \mathrm{Cl} 88.1 \%$ to 96.4\%). If the initial EMR was deemed successful and did not contain submucosal invasion requiring surgery, $98.1 \%(95 \% \mathrm{Cl} 96.6 \%$ to $99.0 \%)$ were adenoma-free and had avoided surgery at 16 months following EMR. Conclusions Following colonic WF-EMR, early recurrent/residual adenoma occurs in 16\%, and is usually unifocal and diminutive. Risk factors were identified. Late recurrence occurs in $4 \%$. Overall, recurrence was managed endoscopically in $93 \%$ of cases. Recurrence is not a significant clinical problem following WF-EMR, as with strict colonoscopic surveillance, it can be managed endoscopically with high success rates.

Trial registration number: NCT01368289.

\section{Significance of this study}

What is already known on this subject?

- Wide-field endoscopic mucosal resection (WF-EMR) is a safe and effective therapy for non-invasive, large, sessile or laterally spreading colonic lesions up to $120 \mathrm{~mm}$ in size.

- Some previous studies of recurrent/residual adenoma following EMR have shown high recurrence rates; however, these studies had significant limitations.

- The optimal timing of surveillance colonoscopy following WF-EMR is unknown.

What are the new findings?

- In a multicentre, prospective study of 1000 consecutive WF-EMRs where the lesion was thought to have been completely treated, early recurrent/residual adenoma (4 months following EMR) was present in $16.0 \%$ and late recurrent/ residual adenoma (16 months following EMR) was uncommon $(4.0 \%)$

- On multivariable analysis, risk factors for recurrent adenoma were lesion size $>40 \mathrm{~mm}$, use of argon plasma coagulation to ablate adenoma and intraprocedural bleeding.

- The recurrent adenoma was usually unifocal and diminutive, and was managed endoscopically in $93.1 \%$ of cases.

- If the initial EMR was deemed successful, and the EMR specimens did not reveal submucosal invasion requiring surgery, then with strict colonoscopy surveillance at 4 and 16 months for further endoscopic treatment as required, $98.1 \%(95 \% \mathrm{Cl} 96.6 \%$ to $99.0 \%)$ were adenoma-free and had avoided surgery at 16 months following EMR. 
Significance of this study

\section{How might it impact on clinical practice in the foreseeable future?}

- Recurrent or residual adenoma following WF-EMR will be treated endoscopically with high rates of success and low likelihood of requiring surgery.

- 4 and 16 months following WF-EMR may be adopted as the appropriate timing for performing surveillance colonoscopies that are critical to achieving a successful outcome.

- EMR has potential to be considered a first-line therapy for large, non-invasive, sessile or laterally spreading colonic lesions, with some advantages over alternatives including surgery or endoscopic submucosal dissection (ESD) that are more resource intensive and carry greater risks of morbidity and mortality.

\section{INTRODUCTION}

Wide-field endoscopic mucosal resection (WF-EMR) performed via colonoscopy is a comparatively new therapy for advanced mucosal neoplasia of the colon. It was proven to be safe and effective for removal of laterally spreading and sessile adenomas up to $100 \mathrm{~mm}$ in size in a large multicentre prospective study. ${ }^{1}$ Large sessile colonic lesions were traditionally managed surgically that remains commonplace. ${ }^{2}$ Endoscopic submucosal dissection (ESD) has been pioneered in the East and is an emerging alternative modality. ${ }^{3}$ WF-EMR is more efficient, less expensive, consumes fewer hospital bed resources (as it is generally performed as a day case) and is associated with less morbidity and mortality than surgery or ESD. ${ }^{5-13}$ WF-EMR has the potential to be accepted as the first-line therapy, but uptake has been limited by concerns that it is associated with high rates (up to $55 \%$ ) of recurrent or residual adenoma at surveillance colonoscopy following successful EMR where the lesion was thought to have been completely treated. ${ }^{14}$

However, the studies on which these concerns were founded were limited by small numbers of patients, smaller lesions, single centre or retrospective designs. ${ }^{14-20}$ Furthermore, recent enhancements in endoscope resolution have facilitated more accurate definition of lesion margins enabling greater confidence regarding the completeness of endoscopic resection and potentially lower recurrence rates. Where recurrent or residual adenoma is present, it is often diminutive and readily treated, and the clinical impact of the recurrence may be minimal. There is no prospective, multicentre, contemporary data available regarding early (3-6 month) and late (>12 month) recurrence following WF-EMR and the clinical significance of this recurrence may have been overstated. In addition, the optimal timing of surveillance colonoscopy following colonic WF-EMR has not yet been determined.

This prospective, multicentre study aimed to quantify recurrent or residual adenoma at colonoscopy performed at 4 months and 16 months following WF-EMR to assess its risk factors and determine its clinical significance.

\section{METHODS}

An ongoing prospective, observational study of all patients referred for WF-EMR of sessile colorectal polyps sized $\geq 20 \mathrm{~mm}$ was conducted at seven Australian academic endoscopy units. Institutional review board approval was obtained at each institution. Consecutive patients were enrolled from July 2008 to July 2012. There were no exclusion criteria. The present study involves long-term follow-up of the original cohort of 479 patients $^{1}$ as well as study of additional consecutive patients until 1000 successful EMRs were achieved.

All lesions were identified at a previous colonoscopy by a nationally accredited consultant endoscopist who referred the patient to the tertiary centre. EMR procedures were performed as day stay only procedures by one of the investigators or a senior therapeutic endoscopy fellow under direct supervision of the investigator. All clinical investigators were gastroenterologists with significant prior colonic EMR experience after training in high-volume tertiary referral centres in Australia or overseas, and recognised as local experts in EMR technique with a wide tertiary referral base. On the day of the procedure, the investigators met with patients and obtained written informed consent. Split-dose bowel preparation was used, but was not otherwise standardised. Intravenous sedation was performed under conscious sedation using a combination of midazolam, fentanyl or propofol. Colonic insufflation was with air, until all centres converted to carbon dioxide insufflation once its advantages were recognised. ${ }^{21}$

Lesions were assessed by white light (high definition once this became available) and narrow band imaging (NBI) without cap attachment. A cap was used in a limited number of cases at the discretion of the endoscopist to optimally manage the specific lesion encountered.

Lesions with features strongly suggestive of submucosal invasion were not attempted for EMR, and the lesion biopsied. The referring endoscopist was contacted and surgical management suggested.

The lesions were removed by 'sequential inject and resect' WF-EMR technique. ${ }^{22-24}$ Normal saline was used as the submucosal injection fluid until January 2010, after which it was replaced with succinylated gelatin (SG) (Gelofusine; B. Braun, Crissier, Switzerland) when SG's technical superiority was proven. $^{25} 26$ The fluid was dyed with indigo carmine. At times this was not available and methylene blue was used as an alternative. Adrenaline 1:100 000 was added to the submucosal injection solution at the discretion of the endoscopist. Injection formed a submucosal fluid cushion to provide a safety zone for snare resection. Resection was predominantly with a 20-mm-sized spiral snare. Following this, where necessary, a small stiff, thin-wired snare was used to resect margins or small areas of residual adenoma. Complete snare excision was the goal in each case. Only when complete snare resection was not possible were ablative techniques used to treat residual adenoma.

Minor residual not amenable to snare excision was treated with diathermy with the snare tip set at 'soft coagulation' (ERBE, VIO 300, Effect 4-6, $80 \mathrm{~W}$ ) or argon plasma coagulation (at 20-40 W and $0.5-1 \mathrm{~L}$ flow depending on lesion location). Visible vessels in the EMR defect that were not bleeding were not routinely treated prophylactically. Similarly, argon plasma coagulation was not used empirically to prophylactically treat the margins of the lesion where complete excision was already thought to have been achieved. Excised tissue was retrieved for histological analysis.

Patients were observed for a minimum of $4 \mathrm{~h}$ following EMR and then discharged on a clear fluid diet overnight with postprocedural instructions.

Detailed data sheets for each patient were completed by the investigators at each site at three time points: (1) immediately postprocedure to describe the EMR procedure and the 
immediate outcomes, (2) at 14 days postprocedure to prospectively detail all complications and histology outcomes and (3) at the time of follow-up colonoscopies. Data storage and analysis was centralised.

Patients with successful endoscopic resection and without submucosal invasive cancer (SMIC) in the WF-EMR specimens underwent colonoscopic surveillance at 4 months (SC1) and 16 months (SC2) after WF-EMR.

The EMR scar was interrogated with white light followed by NBI (Olympus, Tokyo, Japan). The EMR scar was photographed. In cases where there remained some doubt as to the possibility of residual adenoma at the EMR scar, this was biopsied for histology and then treated.

Early recurrence/residual was defined as the presence of any adenoma (microscopic or macroscopic) at the WF-EMR site at SC1. Late recurrence/residual was defined as the endoscopic, photographic and histologic absence of adenoma at SC1, with the presence of adenoma at the site at SC2.

For smaller lesions $(20-25 \mathrm{~mm})$ where en bloc or two-piece excision was achieved, the original study protocol allowed for 12-month surveillance alone to be performed at the discretion of the treating endoscopist. This option was only used in a limited number of cases that are identified separately in the results and excluded from the analyses of early and late recurrence.

If the endoscopic impression at surveillance colonoscopy was that of recurrent/residual adenoma, this was recorded as such and the area treated. Treatment was with excision using a small, stiff, thin-wired snare with electrocautery (ERBE Endocut Q, Effect 3 or Forced coagulation, $25 \mathrm{~W}$ ) and tissue retrieved for histology. If complete snare resection was not possible, the residual adenoma was biopsied and then ablated with either soft coagulation applied via the snare tip (ERBE, Soft coagulation, Effect $4,80 \mathrm{~W}$ ) or argon plasma coagulation (at $20-40 \mathrm{~W}$ and $0.5-1 \mathrm{~L}$ flow depending on lesion location).

All specimens were reviewed by expert gastrointestinal pathologists at the individual centres and cases reviewed at unit academic histology meetings where appropriate.

\section{Statistical analysis}

Results for continuous variables were summarised using mean $(\mathrm{SD})$ or median (IQR) for skewed data. Frequencies (\%) were used to summarise categorical variables and $95 \%$ CI were calculated when relevant. Student $t$ or Mann-Whitney tests were used to compare the distribution of continuous variables by outcome. Pearson's $\chi^{2}$ or Fisher's exact tests were used to test for association between categorical variables and outcome. ORs and their 95\% CI were used to quantify the level of association. Two-tailed tests with a significance level of 5\% were used throughout. All analyses were exploratory and no adjustment was made for multiple comparisons. Multiple logistic regression stratified by study institution was used to identify the independent predictors of outcomes of interest using stepwise variable selection. Candidate variables for inclusion in a model included any variable significant at $\mathrm{p} \leq 0.1$ on univariable analysis. If more than one lesion meeting inclusion criteria was resected during the study period, only the largest lesion was included in the analysis. When analysing risk factors for recurrence of adenoma at follow-up, the continuous variables of lesion size and number of pieces resected were grouped into ordered categories. Statistical analyses were performed using SPSS 22 (IBM SPSS, Armonk, New York, USA).

\section{RESULTS}

\section{Patient and lesion characteristics and EMR success}

Over a 46-month period (July 2008-July 2012), 1219 lesions in 1134 consecutive patients (mean age 68 years (range 27-95, SD 11), $53 \%$ were men) were enrolled. Mean lesion size was $36.4 \mathrm{~mm}$ (range $20-130 \mathrm{~mm}$, SD 17). Median lesion size was $30 \mathrm{~mm}$ (IQR 25-40 mm). EMR was not attempted in 39 patients. This was because the appearance of the lesion was strongly suggestive of SMIC in 25 patients and EMR was not technically possible in 14 . These patients were referred for surgery. EMR was attempted in 1095 cases. In 16 of these attempted cases, the lesion did not elevate with submucosal injection, so these patients were referred for surgery. Single-session complete EMR was unsuccessful in 79 patients, with the reasons detailed in table 1. EMR was successful in 1000 cases.

\section{Early recurrence at $\mathrm{SC} 1$ following successful WF-EMR}

Of the 1000 successful EMR cases, 799 (79.9\%) have undergone SC1. Eighty-eight patients $(8.8 \%)$ were not eligible for colonoscopic surveillance (figure 1). In 113 cases (11.3\%), follow-up was pending. Of the 799 who underwent SC1, there was no recurrent/residual adenoma present in $670(83.9 \%, 95 \%$ CI $81.2 \%$ to $86.3 \%$ ). Early recurrence was present in 128 cases (16.0\%, 95\% CI $13.6 \%$ to $18.7 \%)$. One patient had a perforation during SC1 colonoscope insertion prior to the EMR scar being reached, requiring emergency surgery that was successful, but that patient's recurrence status remains unknown. A flowchart detailing recurrence and outcomes is presented as figure 2 . Biopsies were taken if there was uncertainty as to the possibility of recurrent adenoma. This was done in 116 cases and in only one of those cases did the biopsy histology demonstrate adenoma. This was in a case where multiple clips were applied at the original EMR procedure.

\section{Risk factors for early recurrence}

Univariable analysis of study factor association with recurrence is presented in table 2. Multiple logistic regression analysis (table 3) found that independent predictors of recurrent or residual adenoma were:

(a) increased lesion size compared with a lesion sized $20 \mathrm{~mm}$ : lesion sized $>40 \mathrm{~mm}$ had OR 8.22 (95\% CI 3.90 to 17.3 , $\mathrm{p}<0.001$ ); lesion sized 31-40 mm had OR 3.44 (95\% CI 1.56 to $7.60, \mathrm{p}=0.001)$; lesion sized $21-30 \mathrm{~mm}$ was not significantly different (OR 2.07, 95\% CI 0.93 to $4.57, \mathrm{p}=0.073$ ).

(b) use of argon plasma coagulation (OR 2.42, 95\% CI 1.55 to $3.80, \mathrm{p}<0.001)$.

(c) intraprocedural bleeding (OR 1.66, 95\% CI 1.03 to 2.67, $\mathrm{p}=0.038)$.

\section{Ability to treat early recurrence endoscopically}

At SC1, the recurrent/residual adenoma was easily resected or ablated in 121 of 128 (94.5\%, 95\% CI 89.5\% to 97.6\%). Of

Table 1 Reasons for unsuccessful single-session EMR $(n=79)$

\begin{tabular}{lr}
\hline Submucosal fibrosis & 41 \\
Difficult access or positioning & 24 \\
Deep ileocaecal valve or appendiceal orifice involvement & 5 \\
Deliberate two-stage procedure (large lesion size) & 2 \\
Assessed as high risk for SMIC after commencing EMR & 6 \\
Anaesthetic reaction, procedure abandoned & 1 \\
\hline EMR, endoscopic mucosal resection; SMIC, submucosal invasive cancer.
\end{tabular}




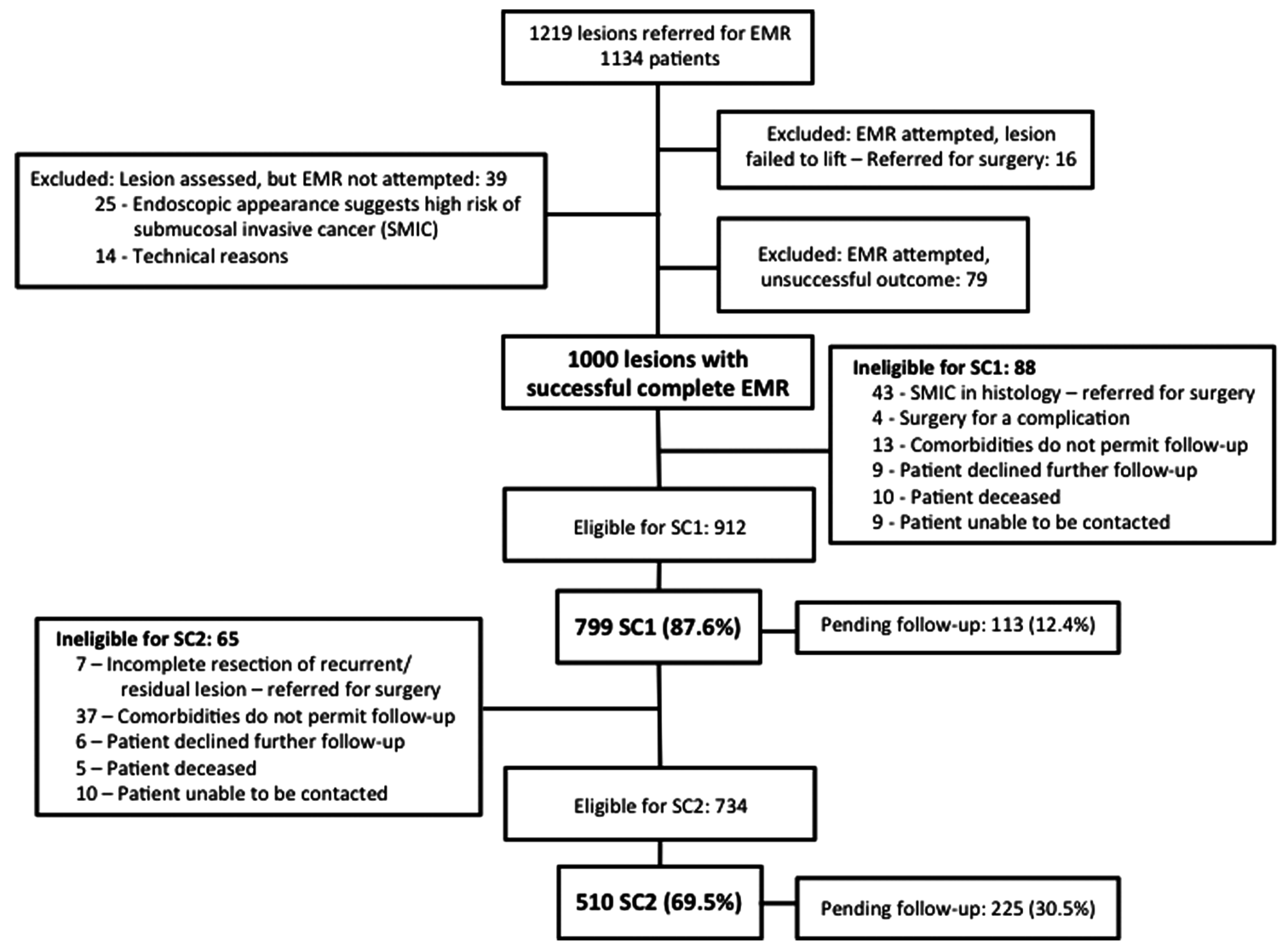

Figure 1 Study enrolment flow diagram.

these 121 patients, 113 had data on the size and morphology of the recurrent/residual adenoma that was unifocal in $76(67.3 \%$, $95 \%$ CI $58.2 \%$ to $75.4 \%)$ and diminutive $(\leq 5 \mathrm{~mm})$ in 81 $(71.7 \%, 95 \%$ CI $62.9 \%$ to $79.4 \%)$ cases. In seven cases, the early recurrent/residual adenoma was not amenable to complete endoscopic resection, for reasons outlined in table 4 . All patients were referred for surgery and all had complete surgical excisions with benign histology and no further treatment required.

\section{Late recurrence at SC2 after normal SC1}

In total, 426 of $670(63.6 \%)$ with no adenoma at SC1 have undergone SC2. There were 17 cases of late recurrence $(4.0 \%$, $95 \%$ CI $2.4 \%$ to $6.2 \%$ ). Sixteen of these cases were completely excised endoscopically; however, one case required surgical management.

\section{Persisting residual adenoma at SC2 following apparent} successful endoscopic management at SC1

Of the 121 patients with early recurrence at SC1 that was managed endoscopically, 84 (69.4\%) have undergone SC2. Persisting residual adenoma was present in 17 cases $(20.2 \%$, $95 \%$ CI $12.7 \%$ to $29.8 \%$ ). In total, 15 of 17 were successfully managed endoscopically and 2 required surgery. Also, 6 of 15 patients have had subsequent colonoscopy 12 months later, all of whom have been clear of residual adenoma. The remaining patients continue to be followed. No cancers were identified during SC1 and SC2 surveillance colonoscopies to date.

\section{Summary of recurrent or residual adenoma results}

In total, recurrence occurred in 145 lesions. This included 128 early recurrences and 17 late recurrences. Endoscopic therapy succeeded in treating recurrence in 135 of 145 cases $(93.1 \%$,
95\% CI $88.1 \%$ to $96.4 \%$ ). Therefore, where a patient's initial EMR was deemed successful, and the EMR specimens did not reveal SMIC requiring surgery, then at 16 months of follow-up, 507 of 517 patients $(98.1 \%$, 95\% CI $96.6 \%$ to $99.0 \%)$ were free of adenoma and had avoided surgery.

\section{Sensitivity analysis of early and late recurrence}

To account for missing data, a sensitivity analysis was performed assuming either half or twice the known recurrence rate. SC1 recurrence in those with available data was $16 \%$. Assuming a rate of $8 \%$ in those with missing data, the overall early recurrence rate would be $14.4 \%$ (95\% CI $12.4 \%$ to $16.7 \%)$. Assuming a rate of $32 \%$ in those with missing data, the overall early recurrence rate would be $19.2 \%$ (95\% CI $16.9 \%$ to $21.8 \%$ ). SC2 recurrence in those with available data was $4.0 \%$. Assuming a rate of $2 \%$ in those with missing data, the overall late recurrence rate would be $3.3 \%$ (95\% CI $2.2 \%$ to $4.9 \%$ ) and assuming a rate of $8 \%$ in those with missing data, the overall late recurrence rate would be $5.5 \%$ (95\% CI $4.0 \%$ to $7.5 \%)$.

\section{Adverse events from treatment of recurrent/residual adenoma}

There were no perforations and no clinically significant episodes of bleeding due to treatment of recurrent/residual adenoma. Unfortunately one patient sustained a perforation on instrument insertion at SC1 that required surgical management.

Intention to treat analysis of successful endoscopic management of the referred lesion

An intention-to-treat analysis of all evaluable patients was performed following SC1 completion to allow the rates of recurrent/residual adenoma recorded in the present study to be 


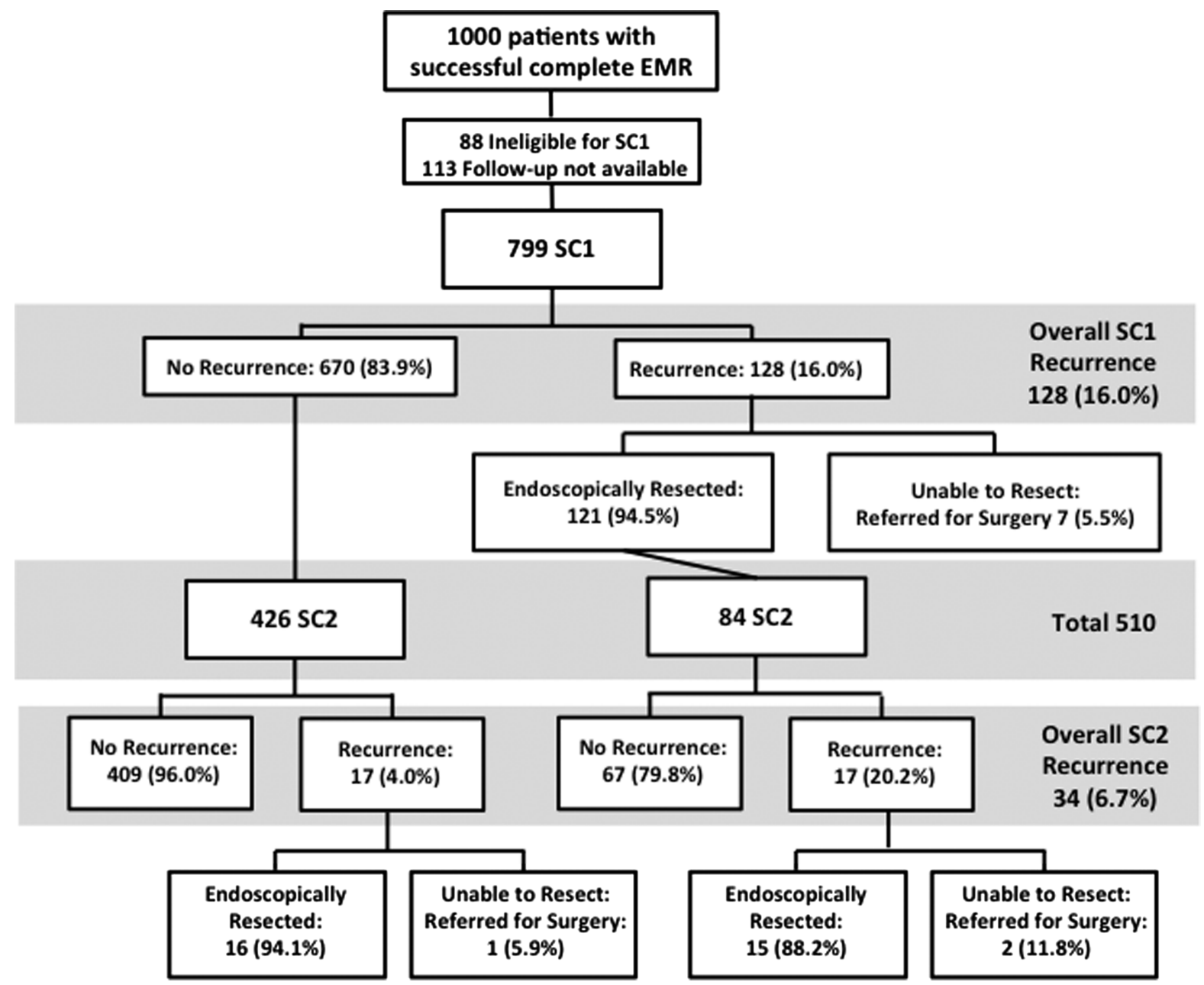

Figure 2 Recurrence flow diagram.

appreciated in the context of the overall cohort and thus estimate overall EMR success.

Of the 1134 patients referred for consideration of EMR, at the conclusion of SC1 procedures performed to date, 157 $(13.8 \%)$ had been referred for surgery. Of these, 149 were referred following the initial EMR procedure for a variety of reasons, including suspected SMIC, technical inability to completely endoscopically resect the lesion, proven SMIC in the EMR specimens or for a perforation adverse event. Seven patients were referred for surgery following SC1 for endoscopically unresectable recurrent adenoma as detailed in table 4. One patient had surgery as a result of colonic perforation during endoscope insertion at SC1. There were four perforations requiring surgery out of 1095 initial EMR procedures, corresponding to a $0.37 \%$ rate of EMR-induced perforation requiring surgery, and with the inclusion of the perforation case at $\mathrm{SC} 1$, a rate of $0.46 \%$ for the overall cohort.

In total, 819 patients had been successfully managed endoscopically at the conclusion of SC1 cases. This included 670 with an initial successful EMR with no recurrence at SC1, 28 patients who had an unsuccessful initial EMR but who were subsequently successfully treated endoscopically and 121 with recurrence at SC1 that was successfully treated endoscopically.

Data were not available at SC1 in 115 patients including 2 patients with unsuccessful initial EMR, and 113 patients with successful initial EMR. Forty-one patients had successful initial EMR procedures, but were ineligible for SC1 for a variety of reasons as detailed in the study enrolment flow diagram (figure 1) and two patients with unsuccessful initial EMR had further therapy planned but were unable to undertake it due to comorbidities. Overall, data were available for 1019 patients (89.9\%) and data at conclusion of SC1 stage were complete in 975 patients. By an intention-to-treat analysis of evaluable patients, endoscopic management at conclusion of SC1 was successful in 819 (84.0\%). At this stage, SC2 data are insufficient to calculate a meaningful intention-to-treat analysis.

\section{DISCUSSION}

We believe that a primary strategy of colonoscopy and WF-EMR performed by an experienced EMR practitioner should be considered a potential first-line therapy for removal of large sessile or laterally spreading adenomas of the colon. It is less expensive, more efficient, consumes fewer hospital resources and is associated with less adverse events than surgery or ESD. ${ }^{5-13}$ However, opponents of EMR cite high rates of recurrent/residual adenoma at surveillance colonoscopy, necessitating further treatment that may not be successful, as their justification for pursuing more invasive strategies. Alternatively, a requirement for 'en bloc' rather than piecemeal resection is claimed.

The present study proves that in a multicentre environment, EMR can achieve low rates of recurrence. Furthermore, when 
Table 2 Univariable analysis of factors associated with recurrence at first surveillance colonoscopy (SC1)

\begin{tabular}{|c|c|c|c|}
\hline & No recurrence & Recurrence & $\mathrm{p}$ Value \\
\hline Age, years (median, SD) & $68.0(11.0)$ & $69.0(10.6)$ & 0.79 \\
\hline \multicolumn{4}{|l|}{ Sex } \\
\hline Male & $348(82.7 \%)$ & $73(17.3 \%)$ & \multirow[t]{2}{*}{0.305} \\
\hline Female & $320(85.3 \%)$ & $55(14.7 \%)$ & \\
\hline \multicolumn{4}{|l|}{ Previous attempt at EMR (n, \%) } \\
\hline Yes & $79(76.7 \%)$ & $24(23.3 \%)$ & \multirow[t]{2}{*}{0.032} \\
\hline No & $590(85.0 \%)$ & $104(15.0 \%)$ & \\
\hline \multicolumn{4}{|l|}{ Lesion size $(\mathrm{n}, \%)$} \\
\hline $20 \mathrm{~mm}$ & $152(95.0 \%)$ & $8(5.0 \%)$ & \multirow[t]{4}{*}{$<0.001$} \\
\hline $21-30 \mathrm{~mm}$ & $230(89.5 \%)$ & $27(10.5 \%)$ & \\
\hline $31-40 \mathrm{~mm}$ & $154(84.6 \%)$ & $28(15.4 \%)$ & \\
\hline$>40 \mathrm{~mm}$ & $132(67.0 \%)$ & $65(33.0 \%)$ & \\
\hline \multicolumn{4}{|l|}{ Location (n, \%) } \\
\hline Rectum $<5 \mathrm{~cm}$ from dentate line & $31(70.5 \%)$ & $13(29.5 \%)$ & \multirow[t]{13}{*}{0.044} \\
\hline Rectum $>5 \mathrm{~cm}$ from dentate line & $90(76.9 \%)$ & $27(23.1 \%)$ & \\
\hline Sigmoid & $67(85.9 \%)$ & $11(14.1 \%)$ & \\
\hline Descending colon & $23(82.1 \%)$ & $5(17.9 \%)$ & \\
\hline Splenic flexure & $13(81.3 \%)$ & $3(18.8 \%)$ & \\
\hline Distal transverse & $22(91.7 \%)$ & $2(8.3 \%)$ & \\
\hline Mid transverse & $30(88.2 \%)$ & $4(11.8 \%)$ & \\
\hline Proximal transverse & $27(90.0 \%)$ & $3(10.0 \%)$ & \\
\hline Hepatic flexure & $58(90.6 \%)$ & $6(9.4 \%)$ & \\
\hline Ascending colon & $157(86.7 \%)$ & $24(13.3 \%)$ & \\
\hline Caecum & $128(86.5 \%)$ & $20(13.5 \%)$ & \\
\hline Caecum ICV involved & $18(72.0 \%)$ & $7(28.0 \%)$ & \\
\hline $\begin{array}{l}\text { Caecum appendiceal orifice } \\
\text { involved }\end{array}$ & $6(66.7 \%)$ & $3(33.3 \%)$ & \\
\hline \multicolumn{4}{|l|}{ Location group $(n, \%)$} \\
\hline Distal colon & $211(79.0 \%)$ & $56(21.0 \%)$ & \multirow[t]{2}{*}{0.007} \\
\hline $\begin{array}{l}\text { Proximal colon (splenic flexure or } \\
\text { above) }\end{array}$ & $459(86.4 \%)$ & $72(13.6 \%)$ & \\
\hline \multicolumn{4}{|l|}{ Paris classification (n, \%) } \\
\hline 0 -Ila & $338(91.4 \%)$ & $32(8.6 \%)$ & \multirow[t]{4}{*}{$<0.001$} \\
\hline $0-\mathrm{ls}$ & $160(78.4 \%)$ & $44(21.6 \%)$ & \\
\hline $0-11 a+l s$ & $127(73.4 \%)$ & $46(26.6 \%)$ & \\
\hline Other & $42(89.4 \%)$ & $5(10.6 \%)$ & \\
\hline \multicolumn{4}{|l|}{ Morphology (n, \%) } \\
\hline Granular & $446(81.2 \%)$ & $103(18.8 \%)$ & \multirow[t]{4}{*}{0.004} \\
\hline Non-granular & $143(91.1 \%)$ & $14(8.9 \%)$ & \\
\hline Mixed & $42(82.4 \%)$ & $9(17.6 \%)$ & \\
\hline Unable to classify & $32(97.0 \%)$ & $1(3.0 \%)$ & \\
\hline \multicolumn{4}{|l|}{ Kudo classification (n, \%) } \\
\hline Kudo I \& II & $78(98.7 \%)$ & $1(1.3 \%)$ & \multirow[t]{4}{*}{$<0.001$} \\
\hline Kudo III & $264(84.9 \%)$ & $47(15.1 \%)$ & \\
\hline Kudo IV & $296(79.1 \%)$ & $78(20.9 \%)$ & \\
\hline Kudo V & $13(92.9 \%)$ & $1(7.1 \%)$ & \\
\hline \multicolumn{4}{|l|}{ Fellow involved $(n, \%)$} \\
\hline Yes & $247(88.2 \%)$ & $33(11.8 \%)$ & \multirow[t]{2}{*}{0.015} \\
\hline No & $420(81.6 \%)$ & $95(18.4 \%)$ & \\
\hline \multicolumn{4}{|l|}{ Intraprocedural bleeding (n, \%) } \\
\hline No & $615(85.5 \%)$ & $104(14.5 \%)$ & $<0.001$ \\
\hline Yes & $55(69.6 \%)$ & $24(30.4 \%)$ & \\
\hline Adrenaline in submucosal injectate ( & & & \\
\hline No & $129(75.0 \%)$ & $43(25.0 \%)$ & $<0.001$ \\
\hline Yes & $540(86.4 \%)$ & $85(13.6 \%)$ & \\
\hline Lifting $(n, \%)$ & & & \\
\hline Lifts well & $625(84.7 \%)$ & $113(15.3 \%)$ & 0.029 \\
\hline Lifts poorly & $42(73.7 \%)$ & $15(26.3 \%)$ & \\
\hline
\end{tabular}

Continued
Table 2 Continued

\begin{tabular}{|c|c|c|c|}
\hline & No recurrence & Recurrence & $\mathrm{p}$ Value \\
\hline \multicolumn{4}{|l|}{ Current type (n, \%) } \\
\hline Non-microprocessor controlled & $80(74.1 \%)$ & $28(25.9 \%)$ & 0.003 \\
\hline Microprocessor controlled & $588(85.5 \%)$ & $100(14.5 \%)$ & \\
\hline \multicolumn{4}{|l|}{ En bloc resection (n, \%) } \\
\hline No & $532(81.2 \%)$ & $123(18.8 \%)$ & $<0.001$ \\
\hline Yes & $138(97.2 \%)$ & $4(2.8 \%)$ & \\
\hline \multicolumn{4}{|l|}{ Additional modality (n, \%) } \\
\hline None & $513(87.5 \%)$ & $73(12.5 \%)$ & $<0.001$ \\
\hline APC & $40(55.6 \%)$ & $32(44.4 \%)$ & \\
\hline Other & $117(83.6 \%)$ & $23(16.4 \%)$ & \\
\hline \multicolumn{4}{|l|}{ APC use $(n, \%)$} \\
\hline No & $630(86.8 \%)$ & $96(13.2 \%)$ & $<0.001$ \\
\hline Yes & $40(55.6 \%)$ & $32(44.4 \%)$ & \\
\hline \multicolumn{4}{|l|}{ Submucosal fibrosis (n, \%) } \\
\hline Yes & $555(85.3 \%)$ & $96(14.7 \%)$ & 0.030 \\
\hline No & $113(77.9 \%)$ & $32(22.1 \%)$ & \\
\hline \multicolumn{4}{|l|}{ Majority polyp histology (n, \%) } \\
\hline Tubular adenoma & $179(87.7 \%)$ & $25(12.3 \%)$ & 0.016 \\
\hline Tubulovillous adenoma & $371(80.8 \%)$ & $88(19.2 \%)$ & \\
\hline Villous adenoma & $15(68.2 \%)$ & $7(31.8 \%)$ & \\
\hline Sessile serrated adenoma/polyp & $89(92.7 \%)$ & $7(7.3 \%)$ & \\
\hline Traditional serrated adenoma & $9(90.0 \%)$ & $1(10.0 \%)$ & \\
\hline $\begin{array}{l}\text { Other (ie, invasive cancer and } \\
\text { underlying polyp histology not } \\
\text { reported, lipoma) }\end{array}$ & $6(100.0 \%)$ & $0(0.0 \%)$ & \\
\hline \multicolumn{4}{|l|}{ Dysplasia (n, \%) } \\
\hline None & $64(94.1 \%)$ & $4(5.9 \%)$ & 0.001 \\
\hline Low grade & $463(85.1 \%)$ & $81(14.9 \%)$ & \\
\hline High grade & $140(76.5 \%)$ & $43(23.5 \%)$ & \\
\hline EMR duration (min) median (SD) & $15.0 \min (17.0)$ & $30.0 \min (24.6)$ & $<0.001$ \\
\hline
\end{tabular}

recurrence is present, it is not a significant clinical problem. The recurrence is usually unifocal, diminutive and easily treated colonoscopically in a single session. Thus, the additional burden of surveillance colonoscopy compared with surgery is minimal, consisting of a single additional colonoscopy. We have shown that if the initial EMR is successful and there is no SMIC in the resected specimens, 98.1\% (95\% CI 96.6\% to 99.0\%) will be free of adenoma and have avoided surgery at 16 months. EMR does not preclude surgery, and for the few cases where recurrence was not manageable endoscopically, surgery was performed.

The low rates of recurrence in our series are consistent with a number of other studies and are likely attributable to a number of factors: (1) we aimed for complete snare excision at the original EMR rather than being satisfied with ablation of residual tissue. Ablation is less controlled and less reliable than excision and also does not provide tissue for histology. (2) Meticulous attention to snare placement was prioritised, aiming to commence at a normal margin, and then work continuously within the submucosal plane, thus avoiding tissue islands. (3) A small (1-2 mm) margin of normal tissue around the lesion was excised, and if required a small stiff thin wire snare was used to achieve this. ${ }^{27}$ (4) Evolution in endoscope technology has significantly enhanced our ability to discern normal from abnormal mucosa, facilitating complete excision. (5) The use of either indigo carmine or methylene blue dye in the submucosal injectate further assists the 
Table 3 Multivariable analysis and best-fitting multiple logistic regression model for factors associated with recurrence at first surveillance colonoscopy (SC1)

\begin{tabular}{llr}
\hline Risk factor for recurrent/residual adenoma & OR & p Value \\
\hline Lesion size & & \\
$\quad 20 \mathrm{~mm}$ & 1 & \\
$21-30 \mathrm{~mm}$ & $2.07(0.93-4.57)$ & 0.073 \\
$31-40 \mathrm{~mm}$ & $3.44(1.56-7.60)$ & 0.002 \\
$>40 \mathrm{~mm}$ & $8.22(3.90-17.3)$ & $<0.001$ \\
Use of argon plasma coagulation (APC) & $2.42(1.55-3.80)$ & $<0.001$ \\
Bleeding during EMR & $1.66(1.03-2.67)$ & 0.038 \\
\hline EMR, endoscopic mucosal resection. & &
\end{tabular}

definition of normal mucosa from adenoma. (6) NBI may be beneficial in detecting the presence of residual adenoma and distinguishing it from normal or cauterised mucosa at the conclusion of the EMR procedure (figure 3 ). ${ }^{27}$

In order to facilitate detection of the EMR scar at surveillance colonoscopy, at the conclusion of the WF-EMR procedure, SPOT tattoo was injected $3 \mathrm{~cm}$ downstream of the resection site. For lesions located in the caecum or ascending colon, careful description of the clock-face location of the lesion with reference to the ileocaecal valve and the number of haustral folds downstream from the valve may also be beneficial. Noting the distance from the anus with a straight scope on withdrawal, or location relative to other anatomical landmarks such as the hepatic flexure or splenic flexure may also be of benefit in facilitating scar detection at surveillance colonoscopy.

The scar from WF-EMR has an obvious and characteristic appearance and is identified as a comparatively pale area with disruption of the usual vascular pattern, often with convergence of surrounding colonic folds. Inspection of the scar with NBI may be helpful to better evaluate the scar for the presence of adenoma.

In some cases, non-specific focal tissue prominence was present at the EMR scar, with the pit pattern not typical of adenoma but doubt remaining. In our experience, this is more common when endoscopic clips have been used to close the EMR site. Where there was a possibility that this tissue was adenomatous, our practice was to biopsy the site, and then treat it, either with resection with a small, stiff, thin-wired snare or ablation. Fibrotic tissue has greater impedance than conventional mucosa and since there is underlying fibrosis at the scar site from the original EMR, the risk of trans-mural injury with superficial treatment of a small area in this manner is low in our experience. Furthermore, the area may be dealt with quickly. Conversely, should the area in doubt be biopsied only, and the

Table 4 Reason for inability to endoscopically manage recurrent/ residual adenoma at SC1 follow-up $(n=7)$

Deep extension into appendiceal orifice (not recognised at initial resection of large lesion)

Deep extension into ileocaecal valve and ileum (not recognised at initial resection of large lesion)

Extensive submucosal fibrosis

Assessed as high risk for SMIC so referred to surgery

SC1, first surveillance colonoscopy performed 4 months after WF-EMR; SMIC, submucosal invasive cancer; WF-EMR, wide-field endoscopic mucosal resection. histology demonstrated adenoma, then the inconvenience of requiring another colonoscopy is high.

Where adenoma was identified at the scar, submucosal injection was not performed in most cases, as the small amount of usually flat residual adenoma does not lift well due to underlying submucosal fibrosis from the initial resection. If injection is performed, it tends to result in elevation of the surrounding normal mucosa, creating a 'canyon effect' that makes resection more difficult. ${ }^{8}$ A 'hot' small, stiff, thin-wired snare with electrocautery was used to resect the adenoma. If the tissue was too small to grasp with snare, this was excised with cold biopsy forceps for histology. The site was then treated with either 'soft coagulation' applied via the snare tip (ERBE, soft coagulation, effect $4,80 \mathrm{~W})$ or argon plasma coagulation. With this approach, we are not relying on the unpredictable depth of tissue injury delivered by a thermal ablative technique, but rather removing all visible neoplastic tissue and then applying thermal ablation as an adjuvant therapy for endoscopically invisible disease.

Previously identified risk factors for early recurrence from the original cohort of 479 patients were lesion size $>40 \mathrm{~mm}$ and the use of argon plasma coagulation (APC) during the original EMR to ablate tissue rather than complete snare excision. ${ }^{1}$ With a significantly larger number of cases in the present series, the findings were similar, but with the strengthening of these risk factors and with the addition of intraprocedural bleeding as an additional independent risk factor. To be recorded as a case of intraprocedural bleeding in the present study, the bleeding had to be sufficient to require therapeutic intervention. Thus, while it is tempting to postulate that recurrence under these circumstances may have resulted from blood obscuring vision of the lesion, since haemostasis was achieved endoscopically in all cases, it is more likely that distraction of the endoscopist from the primary focus of achieving complete resection, to concern about control of haemorrhage, is responsible. This is a reminder that once haemostasis is achieved, particular attention must be paid to thorough inspection of the entire EMR site to assess for residual adenoma. This may be an argument in support of those who advocate for the use of dilute adrenaline in the submucosal injection solution; not to reduce rates of postprocedure bleeding, but rather to attempt to render the EMR field bloodless to enable optimal visibility during EMR.

While the phenomenon of 'late recurrence' after a previous endoscopic and biopsy negative 3-6-month follow-up colonoscopy is recognised, it is uncommon, previously reported in only $2 \%$ of cases. ${ }^{14}$ Our findings are consistent with these data and suggest that if SC1 is clear, then $96 \%$ (95\% CI $93.8 \%$ to $97.6 \%$ ) will be clear at SC2. Thus, while SC2 cannot be abandoned, a normal SC1 is very reassuring. It also suggests that the EMR proceduralist should perform SC1; however, if this is negative, it is reasonable for SC2 to proceed with the referring endoscopist as there is a very low likelihood of intervention being required at the EMR site. This is likely more convenient for patients, particularly when referrals are from rural areas, have advantages for continuity of care and also free up resources at the EMR/academic centres to treat new WF-EMR referrals.

The optimal timing of surveillance colonoscopy following WF-EMR is not proven. Guidelines recommend follow-up colonoscopy at 3-6 months following piecemeal EMR of large lesions. $^{28} 29$ The low proportion of late recurrence in this series would suggest that 4 months is sufficient time for recurrent or residual adenoma to become apparent in most cases. At the same time, where regrowth has occurred, at 4 months the size of the recurrent adenoma is usually still small and thus easily 
Figure 3 Paris classification 0-lla+ls, granular, laterally spreading tumour of the rectosigmoid junction. The lesion is nearly circumferential and extends proximally for $8 \mathrm{~cm}$. Its margins are barely discernible. Endoscopic mucosal resection (EMR) commences at one edge. A margin of normal tissue is included in the resection. The clean edge of the mucosal excision is shown (yellow arrow). The intact muscularis propria layer is visible beneath the defect (red arrow), above which is the indigo carmine dyed, succinylated gelatin (Gelofusine) fluid cushion expanding the submucosa (green arrow). A small amount of adenoma is seen at one edge with the aid of narrow band imaging (blue arrow). This is then excised conservatively to avoid a complete circumferential resection that would cause a stricture. The final result: a wide, almost circumferential mucosal defect without residual adenoma.
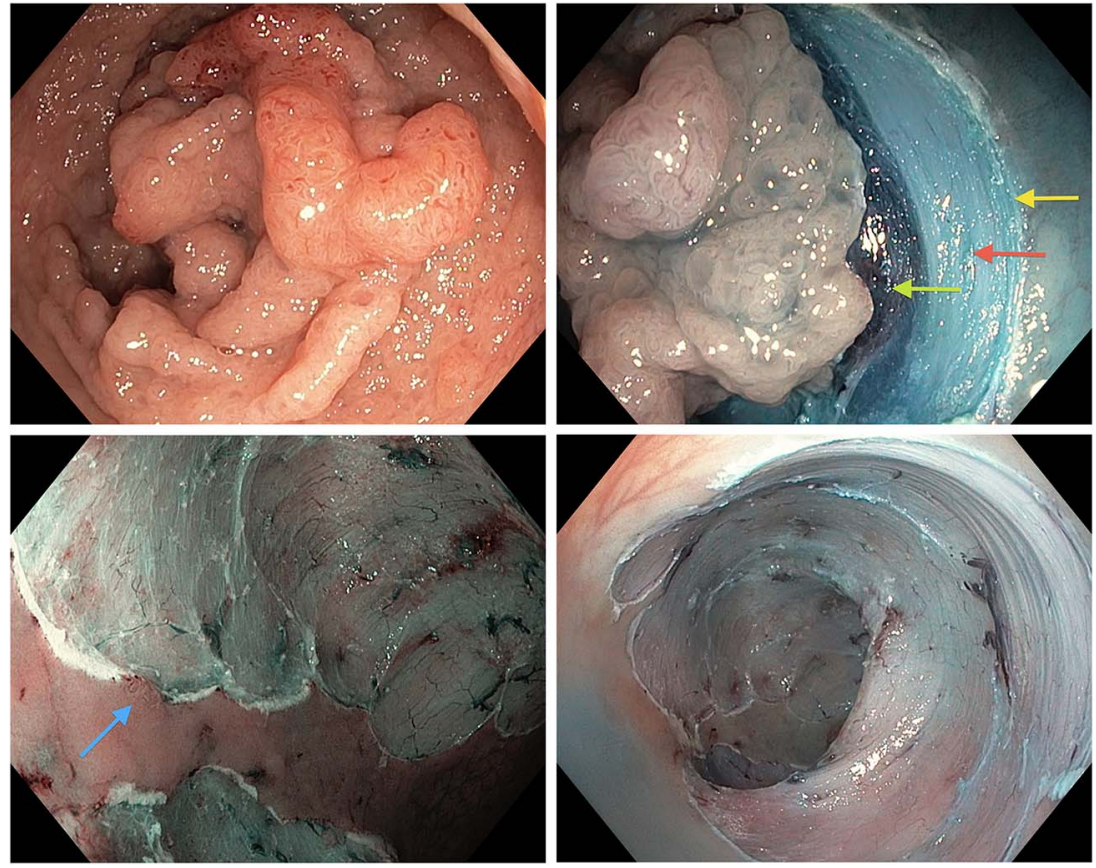

treatable. Based on our experience, we suggest that 4 and 12 months are appropriate intervals for first and second surveillance colonoscopy following WF-EMR. The study of a large number of patients would be required to demonstrate superiority of alternative timing of procedures. However, in some cases, where the procedural risk is high due to significant comorbidities, if the 4-month scope is clear, it may be appropriate to not proceed with the 12-month scope as the risks may outweigh the likelihood of detecting residual adenoma.

There are limitations to our data. We do not yet have complete follow-up data at 16 months post-EMR for all cases. However, given the number of cases studied to date, the results are unlikely to differ significantly with more complete follow-up as demonstrated by the sensitivity analysis. Similarly, while the results at 16 months of follow-up are very encouraging, it would be ideal to have even longer-term follow-up, for example, 3-5 years to further assess longer-term outcomes and prove that EMR provides definitive therapy. We will continue to follow our patients with this goal in mind. Another limitation is the potential for bias inherent in all cohort studies; however, this is limited by the absence of exclusion criteria in the present study.

We believe that our data demonstrate that 'recurrence' is no longer a strong argument that alternative modalities are preferable to WF-EMR for management of advanced colonic mucosal neoplasia. While the Western world grapples with the question of whether to train our endoscopy workforce in ESD, we contend that piecemeal resection by WF-EMR is adequate therapy in the overwhelming majority of cases and that the place for colonic ESD in the West will remain limited. In most Western centres, the burgeoning need for colorectal cancer screening and adenoma surveillance means that 'endoscopy time' is a scarce and limited resource. When the significantly greater costs associated with ESD with respect to procedure duration, postprocedural care, bed occupancy and endoscopic adverse events are considered, we believe that the comparatively inexpensive and efficient EMR procedure should be worthy of consideration as a first-line therapy when performed by experienced EMR practitioners. Furthermore, we believe that the additional costs associated with ESD are difficult to justify for lesions with a low risk of containing carcinoma. In addition, from a training perspective, the barriers to colonic ESD are significant in the Western world, particularly the lack of gastric neoplasia cases on which to gain experience. Moreover, as a snare-based technique, EMR is more readily accessible to most Western endoscopists. The role for ESD is thus limited to lesions with a high risk of containing SMIC, such as those with central depression (Paris classification 0-IIa $+\mathrm{c}$ ) or non-granular surface where there is a greater likelihood of benefit from en bloc resection. However, there are low rates of such lesions among the cohort of patients referred for EMR in our experience, with the vast majority of referred lesions (93\%) not containing submucosal invasion/malignancy. ${ }^{1}$ With even Japanese experts recognising that en bloc resection is not necessary for most laterally spreading tumours (LSTs), ${ }^{30}$ it is likely that only a limited number of ESD experts will be required in most Western cities to manage specific cases referred from institutions in the region. The remainder of the workforce would benefit from training to enhance their EMR skills. Although outcomes of EMR for rectal lesions in the present study were good, being similar to those for more proximal lesions, a European randomised study is currently investigating the optimal therapy for rectal lesions, where proximity to the anus enables other modalities such as transanal endoscopic micro surgery (TEMS) to be compared with EMR.

We conclude that recurrent/residual adenoma following WF-EMR is not a significant clinical issue when patients are carefully followed with mandatory surveillance colonoscopies to allow further endoscopic therapy if required. Early recurrent/ residual adenoma occurs in $16.0 \%$ (95\% CI $13.6 \%$ to $18.7 \%$ ) and is easily treated in the majority. Late recurrence occurs infrequently $(4.0 \%$ (95\% CI $2.4 \%$ to $6.2 \%))$. EMR practitioners should pay careful attention to large lesions $(>40 \mathrm{~mm})$ and resections where intraprocedural bleeding has occurred, and attempt complete snare resection rather than rely on APC ablation of adenoma, as these three risk factors are associated with recurrence at surveillance colonoscopy. There may be a role for empiric/prophylactic APC in the adjuvant setting following perceived complete endoscopic resection, to ablate microscopic foci of adenoma that could not be visualised ${ }^{31} 32$-however, this would require study using the current generation of high- 
definition endoscopes to determine its contemporary utility. When recurrence is detected, endoscopic therapy is successful in 93.1\% (95\% CI $88.1 \%$ to $96.4 \%$ ) of cases. Thus, recurrent or residual adenoma is not a major clinical problem and should not be a reason to look to alternative resource and training intensive techniques.

\section{Author affiliations}

${ }^{1}$ Department of Gastroenterology and Hepatology, Westmead Hospital, Sydney, Australia

${ }^{2}$ Department of Endoscopy, Western Health and The University of Melbourne, Melbourne, Australia

${ }^{3}$ Department of Gastroenterology, The Epworth Hospital, Melbourne, Australia ${ }^{4}$ Department of Gastroenterology, Princess Alexandra Hospital, Brisbane, Australia

${ }^{5}$ Department of Gastroenterology, The Alfred Hospital, Melbourne, Australia

${ }^{6}$ Department of Gastroenterology, Lyell McEwin Hospital, Adelaide, Australia

${ }^{7}$ Westmead Clinical School, University of Sydney, Sydney, New South Wales, Australia

${ }^{8}$ Medical Statistician, Research and Education Network, Westmead Hospital and Sydney University, Sydney, New South Wales, Australia

Contributors AM designed the study, performed procedures, collected data, analysed data, wrote the manuscript and revised the manuscript after review by the coauthors. MJB initiated, designed and led the study, identified and recruited patients, performed procedures, collected data, critically reviewed and finally approved the manuscript. SJW, LFH, GB, WT, RS and SZ identified and recruited patients, performed procedures, collected data and critically reviewed the manuscript. NB assisted significantly with reanalysis of data required for resubmission of the manuscript, and contributed to rewriting of the manuscript. RS collected data and assisted with data analysis. KB performed statistical analysis of data and critically reviewed the manuscript.

Funding The New South Wales Cancer Institution provided funding for a research nurse and data manager to assist with the administration of the study. There was no influence from the institution regarding study design or conduct, data collection, management, analysis or interpretation or preparation, review or approval of the manuscript.

Conflicts of interest None.

Ethics approval Institutional Review Boards at each participating institution.

Provenance and peer review Not commissioned; externally peer reviewed.

\section{REFERENCES}

1 Moss A, Bourke MJ, Williams SJ, et al. Endoscopic mucosal resection outcomes and prediction of submucosal cancer from advanced colonic mucosal neoplasia. Gastroenterology 2011:140:1909-18.

2 Manfredi S, Piette C, Durand G, et al. Colonoscopy results of a French regional FOBT-based colorectal cancer screening program with high compliance. Endoscopy 2008;40:422-7.

3 Tanaka S, Oka S, Kaneko I, et al. Endoscopic submucosal dissection for colorectal neoplasia: possibility of standardization. Gastrointest Endosc 2007;66:100-7.

4 Saito Y, Uraoka T, Matsuda T, et al. Endoscopic treatment of large superficial colorectal tumors: a case series of 200 endoscopic submucosal dissections (with video). Gastrointest Endosc 2007;66:966-73.

5 Ahlenstiel G, Hourigan LF, Brown GJ, et al. Actual endoscopic versus predicted surgical mortality for treatment of advanced mucosal neoplasia of the colon. Gastrointest Endosc 2014;79:119-26.

6 Probst A, Golger D, Anthuber M, et al. Endoscopic submucosal dissection in large sessile lesions of the rectosigmoid: learning curve in a European center. Endoscopy 2012;44:660-7.

7 Bourke MJ. Current status of colonic endoscopic mucosal resection in the West and the interface with endoscopic submucosal dissection. Digestive Endoscopy 2009;21 (Suppl. 1):S27-32.
8 Swan MP, Bourke MJ, Alexander S, et al. Large refractory colonic polyps: is it time to change our practice? A prospective study of the clinical and economic impact of a tertiary referral colonic mucosal resection and polypectomy service (with videos). Gastrointest Endosc 2009:70:1128-36.

9 Onken JE, Friedman JY, Subramanian S, et al. Treatment patterns and costs associated with sessile colorectal polyps. Am J Gastroenterol 2002;97:2896-901.

10 McNicol L, Story DA, Leslie K, et al. Postoperative complications and mortality in older patients having non-cardiac surgery at three Melbourne teaching hospitals. Med J Aust 2007;186:447-52.

11 Birkmeyer JD, Siewers AE, Finlayson EV, et al. Hospital volume and surgical mortality in the United States. N Engl J Med 2002;346:1128-37.

12 Birkmeyer JD, Stukel TA, Siewers AE, et al. Surgeon volume and operative mortality in the United States. N Engl J Med 2003;349:2117-27.

13 Bergman JJ. How to justify endoscopic submucosal dissection in the Western world. Endoscopy 2009;41:988-90.

14 Khashab M, Eid E, Rusche M, et al. Incidence and predictors of "late" recurrences after endoscopic piecemeal resection of large sessile adenomas. Gastrointest Endosc 2009;70:344-9.

15 Buchner AM, Guarner-Argente C, Ginsberg GG. Outcomes of EMR of defiant colorectal lesions directed to an endoscopy referral center. Gastrointest Endosc 2012;76:255-63.

16 Arebi N, Swain D, Suzuki N, et al. Endoscopic mucosal resection of 161 cases of large sessile or flat colorectal polyps. Scand J Gastroenterol 2007;42:859-66.

17 Church JM. Experience in the endoscopic management of large colonic polyps. ANZ J Surg 2003;73:988-95

18 Conio M, Repici A, Demarquay JF, et al. EMR of large sessile colorectal polyps. Gastrointest Endosc 2004;60:234-41.

19 Bergmann U, Beger HG. Endoscopic mucosal resection for advanced non-polypoid colorectal adenoma and early stage carcinoma. Surg Endosc 2003;17:475-9.

20 Doniec JM, Lohnert MS, Schniewind B, et al. Endoscopic removal of large colorectal polyps: prevention of unnecessary surgery? Dis Colon Rectum 2003;46:340-8.

21 Bassan MS, Holt B, Moss A, et al. Carbon dioxide insufflation reduces number of postprocedure admissions after endoscopic resection of large colonic lesions: a prospective cohort study. Gastrointest Endosc 2013;77:90-5.

22 Bourke MJ. Endoscopic Mucosal resection in the colon: a practical Guide. Techniques in Gastrointestinal Endoscopy 2011;13:35-49.

23 Alexander S, Bourke MJ, Williams SJ, et al. EMR of large, sessile, sporadic nonampullary duodenal adenomas: technical aspects and long-term outcome (with videos). Gastrointest Endosc 2009;69:66-73.

24 Hopper AD, Bourke MJ, Williams SJ, et al. Giant laterally spreading tumors of the papilla: endoscopic features, resection technique, and outcome (with videos). Gastrointest Endosc 2010;71:967-75.

25 Moss A, Bourke MJ, Kwan V, et al. Succinylated gelatin substantially increases en bloc resection size in colonic EMR: a randomized, blinded trial in a porcine model. Gastrointest Endosc 2010;71:589-95.

26 Moss A, Bourke MJ, Metz AJ. A randomized, double-blind trial of succinylated gelatin submucosal injection for endoscopic resection of large sessile polyps of the colon. Am J Gastroenterol 2010:105:2375-82.

27 Holt BA, Bourke MJ. Wide field endoscopic resection for advanced colonic mucosal neoplasia: current status and future directions. Clin Gastroenterol Hepatol 2012;10:969-79

28 Repici A, Pellicano R, Strangio G, et al. Endoscopic mucosal resection for early colorectal neoplasia: pathologic basis, procedures, and outcomes. Dis Colon Rectum 2009;52:1502-15.

29 Winawer SJ, Zauber AG, Fletcher RH, et al. Guidelines for colonoscopy surveillance after polypectomy: a consensus update by the US Multi-Society task force on colorectal cancer and the American Cancer Society. Gastroenterology 2006; 130:1872-85.

30 Fujiya M, Moriichi K, Saitoh Y, et al. Endoscopic piecemeal resection is a practical option to cure colorectal tumors. Dig Endosc 2009;21(Suppl 1):S28-30.

31 Brooker JC, Saunders BP, Shah SG, et al. Treatment with argon plasma coagulation reduces recurrence after piecemeal resection of large sessile colonic polyps: a randomized trial and recommendations. Gastrointest Endosc 2002;55:371-5.

32 Elta GH. What is a defiant polyp and how good are we at removing them? Gastrointest Endosc 2012;76:264-6. 